\title{
Effect of Gravity Modulation on the Onset of Rayleigh-Bénard Convection in a Weak Electrically Conducting Couple Stress Fluid with Saturated Porous Layer
}

\author{
Sameena Tarannum ${ }^{1}$ \\ ${ }^{1}$ Department of Professional Studies, \\ Christ University, Bangalore, \\ India.
}

\author{
S. Pranesh ${ }^{2}$ \\ ${ }^{2}$ Department of Mathematics, \\ Christ University, Bangalore, \\ India.
}

\begin{abstract}
The effect of time-periodic body force (TBF, also called gravity modulation) of small amplitude in a weak electrically conducting couple stress fluid with saturated porous layer is investigated by using a linear stability analysis. A regular perturbation method is used to arrive at an expression for the correction Rayleigh number. The Venezian approach is adopted in arriving at the critical Rayleigh and wave number for small amplitudes of TBF. The effect of roles of Couple stress parameter, Hartmann number, Darcy number, Porous parameter and Prandtl number on the onset of convection is studied. It is found that TBF leads to delay in convection. Also the results suggest that instead of taking electrically non-conducting fluid it is better to consider electrically conducting fluid with weak electrical conductivity as this ensures a stable environment in the presence of a magnetic field. The system is most stable with respect to TBF.
\end{abstract}

Keywords: Gravity modulation, Couple stress fluid, Porous medium, Darcy number, Couple stress parameter, Hartmann number.

\section{INTRODUCTION}

The Rayleigh- Bénard problem in its simplest form and one that was the earliest to be investigated is the so called infinite layer case. In such a case, a layer of fluid is constrained between two infinite horizontal surfaces. The surface is heated from below and cooled from above, i.e., the lower surface is at a higher temperature than the upper surface. The heated from below is said to have an adverse temperature gradient because the fluid at the bottom will be lighter than the fluid at the top and this top-heavy arrangement is potentially unstable. When the temperature gradient is below a certain value, the natural tendency of the fluid to move, because of buoyancy, will be inhibited by its own viscosity and thermal diffusivity. Thus the thermal instability will manifest itself only when the adverse temperature gradient exceeds a certain critical value.

Convection instability in a horizontal porous layer with uniform temperature gradient has been investigated extensively by several authors using Darcy model because of its relevance to variety of situations in science and engineering problems. The existence of a fluid layer adjacent to a layer of fluid-saturated porous medium is a common occurrence in both the natural (geophysical) and industrial environments, including such engineering applications as solar collector with a porous absorber, journal bearings, fibrous and granular insulation where the insulation occupies only part of the space separating the heated and cooled walls, etc. The applications also include underground spread of pollutants, convection in earth's crust, geothermal energy, extraction of ceramic processing solid, matrix heat exchanger, food storage, porous heat exchangers, etc.

By a porous medium we mean a material consisting of a solid matrix with interconnected void. We suppose that the solid matrix is either rigid or it undergoes small deformation. The interconnectedness of the void (the pores) allows the flow of one or more fluids through the material. In the simplest situation ("single-phase flow") the void is saturated by a single fluid. In "two-phase flow" a liquid and a gas share the void space.

Typical studies in this field of flow through and over porous layers consider the porous medium to have constant porosity and permeability. However, two situations arise in this connection:

1) In the presence of a solid boundary, there is a need to redefine porosity and permeability due to the increase in permeability and porosity near a solid wall, thus giving rise to channelling effects. Channelling effects have an impact on shear stress and heat transfer near the wall.

2) In flow over porous layers, there exists a permeability discontinuity at the interface between the flow regions. This has an impact on momentum and heat transfer across the interface.

An important class of fluid differs from that of Newtonian fluids, in that the relationship between the shear stress and flow field is more complicated. Such fluids are non-Newtonian. The study of non-Newtonian fluids has attracted much attention, because of their practical applications in industry particularly in extraction of crude oil from petroleum products, applications in biology, physiology and technology. In addition, the effects of heat 
and mass transfer in non-Newtonian fluid also have great importance in engineering applications like the thermal design of industrial equipment dealing with molten plastics, polymeric liquids, foodstuffs, or slurries. Several investigators have extended many of the available convection heat and mass transfer problems to include the non-Newtonian effects. In the category of non-Newtonian fluids, couple stress has distinct features, such as polar effect in addition to possessing large viscosity. The consideration of couple stress in addition to classical Cauchy stress, has led to the recent development of several theories of fluid micro continua. One such couple stress theory of fluids was developed by Stokes (1966) and represents the simplest generalization of the classical theory which allows for polar effects such as the presence of couple stresses and body couples.

Couple stress in the consequence of assuming that mechanical action of one part of a body on another across a surface is equivalent to a force and moment distribution. In the classical non-polar theory, moment distribution is not considered and mechanical action is assumed to be equivalent to the force distribution only. One of the applications of couple-stresses in fluid is its use in the study of the mechanism of lubrication of synovial joints, which has become the objective of scientific research. The study of couple stress fluid flows has been the subject of great interest due to its widespread and scientific applications. Few authors P.G. Siddeshwar and S. Pranesh (2004), Veera Krishna et al. (2010) and I.S. Shivakumara (2012) have considered couple stress fluid in investigating Rayleigh- Bénard convection under different situations.

Couple stress fluid theory developed by Stokes (1966), is one among the polar fluid theories which considers couple stresses in addition to the classical Cauchy stress. It is the simplest generalization of the classical theory of fluids which allows for polar effects such as the presence of couple stresses and body couples. This fluid theory is discussed in detail by Stokes (1984) in his treatise "Theories of fluids with microstructure" where in he also presented a list of problems discussed by researchers with reference to this theory.

Another important class of natural convection problem is concerned with the difficulty in avoiding the convection in the earth's gravitational field even when the basic temperature gradient is uniform and interfacial instabilities can be ignored. Many theoretical and experimental studies dealing with material processing or physics of fluids under the microgravity condition aboard an orbiting spacecraft have been carried out in recent years. Owing to several unavoidable sources of residual acceleration experienced by a spacecraft, the gravity field in an orbiting laboratory is not constant in a microgravity environment, but is a randomly fluctuating field. This fluctuating gravity is referred to as g-jitter. It is reported in the literature that vibrations can either substantially enhance or retard heat transfer and thus drastically affect the convection.

The effect of gravity modulation on a convection stable configuration can significantly influence the stability of a system by increasing or decreasing its susceptibility to convection. In general, a distribution of stratifying agency that is convectively stable under constant gravity conditions can be destabilized when a time-dependent component of the gravity field is introduced certain combinations of thermal gradients, physical properties and modulation parameters may lead to parametric resonance and hence, to the stability of the system. P.G. Siddheshwar and S. Pranesh (1999, 2000), Malashetty and Basavaraja (2002), P.G. Siddheshwar and Abraham (2003) and recently by S. Pranesh et al (2014) have studied on the onset of convection in Newtonian and non-Newtonian fluids.

With the above motivation, the main objective of this paper is to study the effects of fluctuating gravity in a weak electrically conducting couple stress fluid with a saturated porous layer on the onset of Rayleigh- Bénard convection.

\section{MATHEMATICAL FORMULATION}

Consider a layer of a weak electrically conducting couple stress fluid with saturated porous layer confined between two infinite horizontal walls distant ' $d$ ' apart (fig 1). The uniform magnetic field is directed along the z-axis. A Cartesian co-ordinate system is taken with origin in the lower boundary and z-axis vertically upwards.

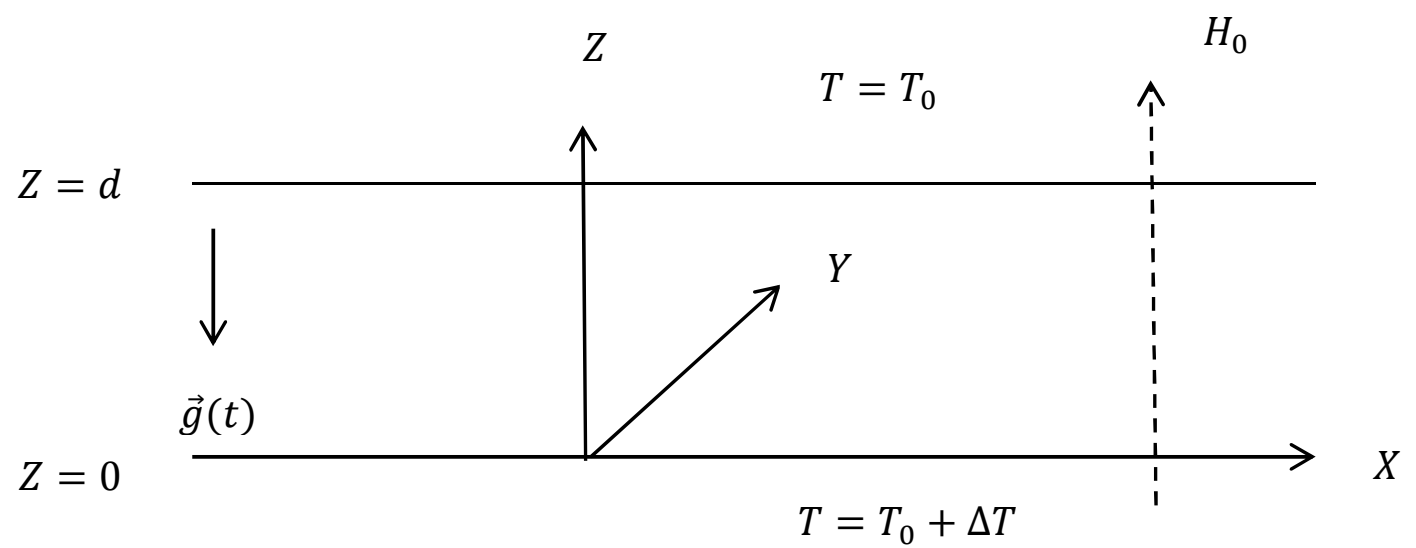

Fig 1: Physical configuration of the problem 
The governing equations are:

Continuity equation:

$$
\nabla \cdot \vec{q}=0
$$

\section{Conservation of Linear Momentum:}

$\rho_{0}\left[\frac{1}{\epsilon} \frac{\partial \vec{q}}{\partial t}+\frac{1}{\epsilon^{2}}(\vec{q} \cdot \nabla) \vec{q}\right]=-\nabla p+\rho \vec{g}(t)+\frac{\mu^{\prime}}{k} \nabla^{2} \vec{q}-\frac{\mu_{e}}{k} \vec{q}+$ $\vec{J} \times \vec{B}$,

$$
\vec{g}(t)=g_{0}[1+\delta \cos \gamma t] \hat{k},
$$

Conservation of energy:

$$
\gamma^{\prime} \frac{\partial T}{\partial t}+(\vec{q} \cdot \nabla) T=\chi \nabla^{2} T
$$

Equation of state:

$$
\rho=\rho_{0}\left[1-\alpha\left(T-T_{0}\right)\right],
$$

Constitutive equations:

$$
\begin{aligned}
& \vec{J}=\sigma(\vec{q} \times \vec{B}), \\
& \vec{B}=\mu_{m} \vec{H},
\end{aligned}
$$

where $\vec{q}$ is the velocity, $\rho_{0}$ is the constant density, $p$ is the pressure, $\rho$ is the density, $\vec{g}$ is the gravitational force, $\in$ is the porosity, $\mu^{\prime}$ is the couple stress viscosity, $\mu_{e}$ is the effective viscosity, $\chi$ is the thermal conductivity, $\gamma^{\prime}$ is the ratio of heat capacity, $\vec{J}$ is the current density, $\vec{B}$ is the magnetic induction vector, $\mathrm{k}$ is the permeability of the porous media, $g_{0}$ is the mean gravity, $\delta$ is the small amplitude of gravity modulation, $\gamma$ is the frequency, $\mathrm{t}$ is the time, $T$ is the temperature, $\alpha$ is the coefficient of thermal expansion, $\sigma$ is the magnetic conductivity, $\mu_{m}$ is the magnetic permeability, $\vec{H}$ is the magnetic field.

For a weak electrically conducting fluid the Lorentz force $\vec{J} \times \vec{B}$ on using the constitutive equations (6) and (7) can be written as

$$
\vec{J} \times \vec{B}=-\mu_{m}^{2} \sigma H_{0}^{2} \vec{q}
$$

where $H_{0}$ is the applied transverse magnetic field.

\section{BASIC STATE}

The basic state of the fluid is quiescent and is described by

$\vec{q}=\vec{q}_{b}(0,0,0), \rho=\rho_{b}(z), T=T_{b}(z), p=p_{b}(z)$.

The temperature $T_{b}$, pressure $p_{b}$ and density $\rho_{b}$ satisfy

$$
\begin{aligned}
& \gamma^{\prime} \frac{\partial T_{b}}{\partial t}=\chi \frac{\partial^{2} T_{b}}{\partial z^{2}} \\
& \frac{\partial p_{b}}{\partial z}=-\rho_{b} g_{0}[1+\delta \cos \gamma t] \hat{k}, \\
& \rho_{b}=\rho_{0}\left[1-\alpha\left(T_{b}-T_{0}\right)\right] .
\end{aligned}
$$

\section{LINEAR STABILITY ANALYSIS}

Let the basis state be disturbed by an infinitesimal thermal perturbation. We now have

$\vec{q}=\vec{q}_{b}+\vec{q}^{\prime}, \rho=\rho_{b}(z)+\rho^{\prime}, T=T_{b}(z)+T^{\prime}, p=p_{b}(z)+p^{\prime}$.

The prime indicates that the quantities are infinitesimal perturbations.

Substituting equation (13) into equations (1) to (5), using equation (8) in equation (2) and using the basic state solution, we get linearized equations governing the infinitesimal perturbations in the form

$$
\nabla \cdot \vec{q}^{\prime}=0
$$

$\frac{\rho_{0}}{\epsilon} \frac{\partial \vec{q}^{\prime}}{\partial t}=-\nabla p^{\prime}-\rho^{\prime}[1+\delta \cos \gamma t] g_{0} \hat{k}+\frac{\mu^{\prime}}{k} \nabla^{2} \vec{q}^{\prime}-\frac{\mu_{e}}{k} \vec{q}^{\prime}-$ $\mu_{m}^{2} \sigma H_{0}^{2} \vec{q}^{\prime}$,

$$
\begin{aligned}
& \gamma^{\prime} \frac{\partial T^{\prime}}{\partial t}=\frac{\Delta T}{d} w^{\prime}+\chi \nabla^{2} T^{\prime}, \\
& \rho^{\prime}=-\rho_{0} \alpha T^{\prime} .
\end{aligned}
$$

Operating curl twice to eliminate pressure term on equation (15) and using (17), we get

$$
\begin{aligned}
& \frac{\rho_{0}}{\epsilon} \frac{\partial}{\partial t}\left(\nabla^{2} w^{\prime}\right)=\rho_{0} \alpha[1+\delta \cos \gamma t] g_{0} \nabla_{1}^{2} T^{\prime}+\frac{\mu^{\prime}}{k} \nabla^{4} w^{\prime}- \\
& \frac{\mu_{e}}{k} \nabla^{2} w^{\prime}-\mu_{m}^{2} \sigma H_{0}^{2} \nabla^{2} w^{\prime} .
\end{aligned}
$$

The perturbation equations (14) and (16) to (18) are nondimensionalized by the following definition

$$
\left(x^{*}, y^{*}, z^{*}\right)=\left(\frac{x}{d}, \frac{y}{d}, \frac{z}{d}\right), t^{*}=\frac{t}{d^{2} \in / k}, w^{*}=\frac{w^{\prime}}{\chi / d}, T^{*}=\frac{T^{\prime}}{\Delta T}
$$

The perturbation equations (14) and (16) to (18) on using (19), we get

$\frac{1}{P r} \frac{\partial}{\partial t}\left(\nabla^{2} w\right)=R[1+\delta \cos \Omega t] \nabla_{1}^{2} T+C \nabla^{4} w-\frac{1}{D a} \nabla^{2} w-$ $M^{2} \nabla^{2} w$

$$
\left(M_{1} \frac{\partial}{\partial t}-\nabla^{2}\right) T=w,
$$

where the asterisks have been dropped for simplicity and the non-dimensional parameters $\operatorname{Pr}, R, C, D a, M^{2}, M_{1}$ and $\Omega$ are given as

$$
\begin{array}{ll}
\operatorname{Pr}=\frac{\mu_{e} \epsilon^{2}}{\rho_{0} \chi} & \text { (Prandtl number), } \\
R=\frac{\rho_{0} \alpha g_{0} \Delta T d^{3}}{\mu_{e} \chi} & \text { (Rayleigh number), } \\
C=\frac{\mu^{\prime}}{\mu_{e} k} & \text { (Couple stress parameter), } \\
D a=\frac{k}{d^{2}} & \text { (Darcy number), } \\
M^{2}=\frac{\mu_{m}^{2} \sigma H_{0}^{2} d^{2}}{\mu_{e}} & \text { (Hartmann number), } \\
M_{1}=\frac{\gamma^{\prime}}{\epsilon} & \text { (Porous parameter) and } \\
\Omega=\frac{d^{2} \epsilon \gamma}{\chi} & \text { (Non-dimensional modulation frequency). }
\end{array}
$$

Equations (20) and (21) are solved subject to the conditions

$$
w=D^{2} w=T=0 \text { at } z=0,1 \text {. }
$$

Eliminating $w$ from equations (20) and (21), we get an equation for $T$ in the form

$$
\begin{aligned}
& \left(\frac{1}{P r} \frac{\partial}{\partial t} \nabla^{2}-C \nabla^{4}+\frac{1}{D a} \nabla^{2}+M^{2} \nabla^{2}\right)\left(M_{1} \frac{\partial}{\partial t}-\nabla^{2}\right) T= \\
& R[1+\delta \cos \Omega t] \nabla_{1}^{2} T .
\end{aligned}
$$

In dimensionless form, the velocity boundary conditions for solving equation (23) are obtained from equations (20) to $(22)$ in the form

$$
T=\frac{\partial^{2} T}{\partial z^{2}}=\frac{\partial^{4} T}{\partial z^{4}}=0 \text { at } z=0,1 .
$$




\subsection{STABILITY ANALYSIS}

We now seek the eigen-function $T$ and eigen-values $R$ of the equation (23) in the form

$(R, T)=\left(R_{0}, T_{0}\right)+\delta\left(R_{1}, T_{1}\right)+\delta^{2}\left(R_{2}, T_{2}\right)+$.

The expansion of (25) is substituted into equation (23) and the coefficients of various powers of $\delta$ are equated on either side of the equation. The resulting system of equation is

$L T_{0}=0$

$L T_{1}=\left(R_{1}+R_{0} f\right) \nabla_{1}^{2} T_{0}$,

$L T_{2}=\left(R_{1}+R_{0} f\right) \nabla_{1}^{2} T_{1}+\left(R_{2}+R_{1} f\right) \nabla_{1}^{2} T_{0}$,

where

$L=\left[\left(\frac{1}{P r} \frac{\partial}{\partial t} \nabla^{2}-C \nabla^{4}+\frac{1}{D a} \nabla^{2}+M^{2} \nabla^{2}\right)\left(M_{1} \frac{\partial}{\partial t}-\nabla^{2}\right)-\right.$ $R_{0} \nabla_{1}^{2}$.

Each of $T_{n}$ is required to satisfy the boundary condition (24).

The marginally stable solution of the problem is the general solution of the equation (26), i.e.

$$
T_{0}=\sin \pi z e^{i(l x+m y)} .
$$

corresponding to the lowest mode of convection with the corresponding eigen value

$$
R_{0}=\frac{k_{1}^{2}\left(\eta+M^{2}\right)}{a^{2}},
$$

where

$$
\begin{aligned}
& \eta=1+C k_{1}^{2}, \\
& k_{1}^{2}=\pi^{2}+a^{2}, \\
& a^{2}=l^{2}+m^{2} .
\end{aligned}
$$

Equation (27) on using equation (30) becomes

$$
L T_{1}=-R_{1} a^{2} \sin \pi z-R_{0} a^{2} f \sin \pi z .
$$

If the above equation is to have a solution, the right hand side must be orthogonal to the null-space of the operator L. This implies that the time independent part of the RHS of the equation (32) must be orthogonal to $\sin \pi z$. Since $f$ varies sinusoidal with time, the only steady term on the RHS of equation (32) is $-R_{0} a^{2} f \sin \pi z$ so that $\mathrm{R}_{1}=0$.

It follows that all the odd coefficients i.e. $\mathrm{R}_{1}=\mathrm{R}_{3}=\ldots \ldots=0$ in equation (25).

Using equation (29), we find that

$$
L\left[\sin \pi z e^{i(l x+m y-\Omega \mathrm{t})}\right]=L(\Omega) \sin \pi z e^{i(l x+m y-\Omega \mathrm{t})},
$$
where

$$
\begin{aligned}
& L(\Omega)=Y_{1}+i Y_{2}, \\
& Y_{1}=\left[\frac{\Omega^{2} M_{1} k_{1}^{2}}{P r}-\left(k_{1}^{2}\right)^{2} X_{1}\right]+R_{0} a^{2}, \\
& Y_{2}=\Omega\left[\frac{\left(k_{1}^{2}\right)^{2}}{P r}+M_{1} k_{1}^{2} X_{1}\right], \\
& X_{1}=\eta+M^{2}, \\
& \eta=1+C k_{1}^{2}, \\
& k_{1}^{2}=\pi^{2}+a^{2}, \\
& a^{2}=l^{2}+m^{2} .
\end{aligned}
$$

The particular solution of equation (32) is

$T_{1}=\frac{1}{|L(\Omega)|^{2}}\left(Y_{1} \cos \Omega \mathrm{t}-Y_{2} \sin \Omega \mathrm{t}\right)\left(-R_{0} a^{2} \sin \pi z\right)$.
The solution of the homogeneous equation corresponding to equation (32) involves a term proportional to $\sin \pi z$. However, addition of such a term to a complete solution of equation (32) merely amounts to a renormalization of $T$ because all the terms proportional to $\sin \pi z$ can then be grouped to define a new $T_{0}$ with corresponding $T_{1}, T_{2}, \ldots$. Hence, we can assume that $T_{0}$ is orthogonal to all other $T_{n}$ 's.

The equation of $T_{2}$ is

$$
L T_{2}=-R_{0} f a^{2} T_{1}-R_{2} a^{2} T_{0},
$$

We shall not solve equation (35), but will use this to determine $R_{2}$. For the existence of a solution of equation (35), it is necessary that the steady part of its right hand side is orthogonal to $\sin \pi z$. This gives,

$-\int_{0}^{1} R_{0} f a^{2} T_{1} \sin \pi z d z-\int_{0}^{1} R_{2} a^{2} T_{0} \sin \pi z d z=0$, Taking time average, we get,

and finally

$$
R_{2}=-2 R_{0} \int_{0}^{1} \overline{f T_{1}} \sin \pi z d z,
$$

$$
R_{2}=\frac{R_{0} a^{2} Y_{1}}{2|L(\Omega)|^{2}} .
$$

\section{RESULTS AND DISCUSSION}

In this paper we have made an analytical study of the effect of gravity modulation on the onset of convection in a weak electrically conducting couple stress fluid with saturated porous layer. As per the stated motivation on control of convection, the following effects on the classical Rayleigh- Bénard problem are considered:

(i) Inhibition of convection by suspended particles, and

(ii) Gravity modulation.

These two effects are, respectively, represented by the couple stress parameter $C$ and $\vec{g}(t)$. Before embarking on a discussion of the results depicted by the Figs. 2-10, we note that oscillatory mode of convection does not come into picture in couple stress fluids. It should be noted that gravity modulation affects the entire bulk of fluid between the boundary plates.

The analysis presented in this paper is based on the assumption that the amplitude of the gravity modulating is small. The validity of the results obtained here depends on the value of the modulating frequency $\Omega$. When $\Omega<1$, the period of modulation is large. The gravity modulation affects the entire volume of the fluid, resulting in the growth of the disturbance. On the other hand, the effect of modulation disappears for the large frequency. This is due to the fact that the buoyancy force takes a mean value leading to equilibrium state of the unmodulated case. In view of this, we choose only moderate value of $\Omega$ in our study. It must be noted here that because of the presence of suspended particles in the fluid and according to Einstein's relation for viscosity, the value of Prandtl number is taken higher than those of clean fluid.

Figure 2 is the plot of correction Rayleigh number $R_{2 C}$ versus frequency of modulation $\Omega$ for different values 
of couple stress parameter $C$. In the figure we observe that as $\mathrm{C}$ increases, $R_{2 C}$ also increases and thus stabilizes the system. $\mathrm{C}$ is the indicative of the concentration of the suspended particles. The physical reason for the nature of effect of $C$ on $R_{2 C}$ can be given by invoking the Einstein law on viscosity of suspensions. Einstein relation for suspended particles $\mu=\mu_{0}(1+2.5 \alpha \phi)$, where $\mu$ and $\mu_{0}$ are the viscosities of suspension(i.e. clean fluid + suspended particles) and clean fluid respectively, $\alpha$ is the shape factor and $\phi$ is the volume fraction of the suspended particles. The shape factor $\alpha$ is 1 for spherical particles and greater than 1 for other shapes such as ellipsoids, rods, etc. In order to understand an increase in the viscosity of a suspension with concentration, it is helpful to realize that the viscosity of any fluid is associated with dissipation of mechanical energy into heat within the fluid. The viscosity of a suspension also depends on various other factors such as viscosity of the carrier liquid (suspending medium), the interaction between the particles, on whether the particles are rigid or deformable and on the temperature and electrical conductivity. We keep the analysis to the bare minimum and do not venture into delineating the above individual contributions to viscosity. We just assume that the viscosity of a suspension is higher than that of the carrier liquid. The assumed viscosity concentration relation is valid for low concentrations. In the case of higher concentrations one will have to add quadratic term in $\phi$ to the Einstein relation. In the light of the above discussion, we consider values of Prandtl number of fluids with suspended particles to be higher than those of the fluids without suspended particle.

Figure 3 is the plot of $R_{2 C}$ versus $\Omega$ for different values of Hartmann number $M^{2}$. We observe from the figure that increase in $M^{2}$, increases $R_{2 C}$. In making conclusions from the figure, we should also note that $\mathrm{C}$ and
$M^{2}$ influence $R_{2 C}$. We find that $R_{2 C}$ increases with increase in $\mathrm{C}$ and $M^{2}$. When the magnetic field strength increases, it induces viscosity into the fluid, and the magnetic lines are distorted by convection. Then these magnetic lines hinder the growth of disturbances, leading to the delay in the onset of instability.

Figure 4 is the plot of $R_{2 C}$ versus $\Omega$ for different values of Darcy number $D a$. We observe that increase in $D a$, decreases $R_{2 C}$ and thus destabilize the system.

Figure 5 is the plot of $R_{2 C}$ versus $\Omega$ for different values of Porous parameter $M_{1}$. In the figure we observe that as $M_{1}$ increases, $R_{2 C}$ decreases and thus destabilize the system.

Figure 6 is the plot of $R_{2 C}$ versus $\Omega$ for different values of Prandtl number $P r$. We observe that increase in $P r$, increases $R_{2 C}$. We can infer from this that the effect of increase in the concentration of the suspended particles is to stabilize the system. It is appropriate to note that $\mathrm{Pr}$ does not affect the $R_{0}$-part of $R$, it effects only $R_{2}$.

Figures 7, 8, 9 and 10 are the plots of $R_{2 C}$ verses $\Omega$ for different values of $C, D a, M_{1}$ and $P r$ respectively for $M^{2}=0$. The effects of these parameters are quantitatively similar to that of the results for $M^{2} \neq 0$.

Comparing our results for $C=0$ with the results of P.G. Siddheshwar and S. Pranesh (2000) for $N_{1}=0$, we found that the critical Rayleigh number in the case of finitely conducting fluid is less than that of weak electrically conducting fluid. This is true for the fluids with suspended particles. These observed results have been analysis bearing in mind that in the case of weak electrically conducting fluids, the resistance to flow is higher compared to the finitely electrically conducting case. 


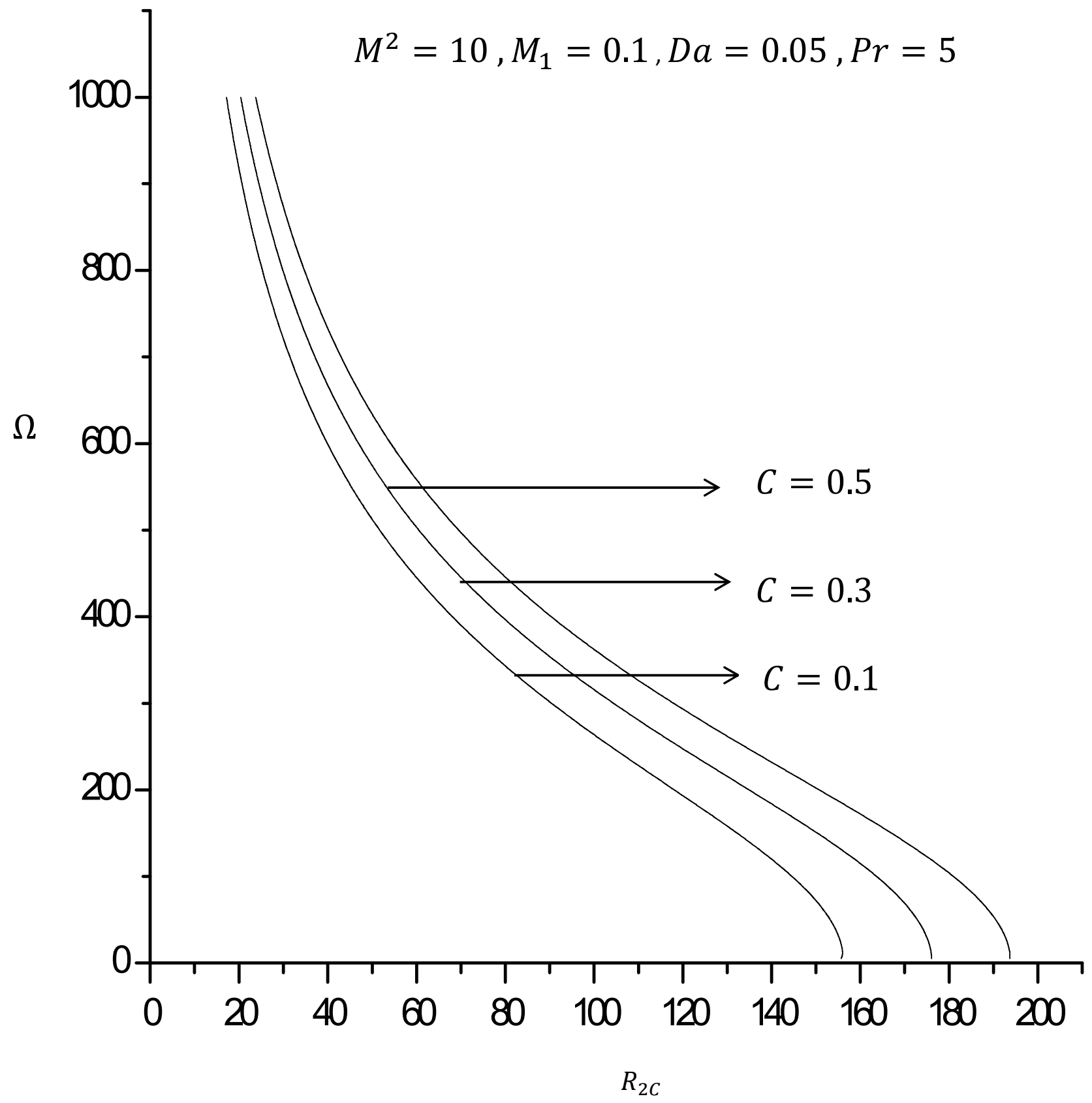

Fig 2: The plot of correction Rayleigh number $R_{2 C}$ vs frequency of modulation $\Omega$ for different values of Couple stress parameter $C$. 


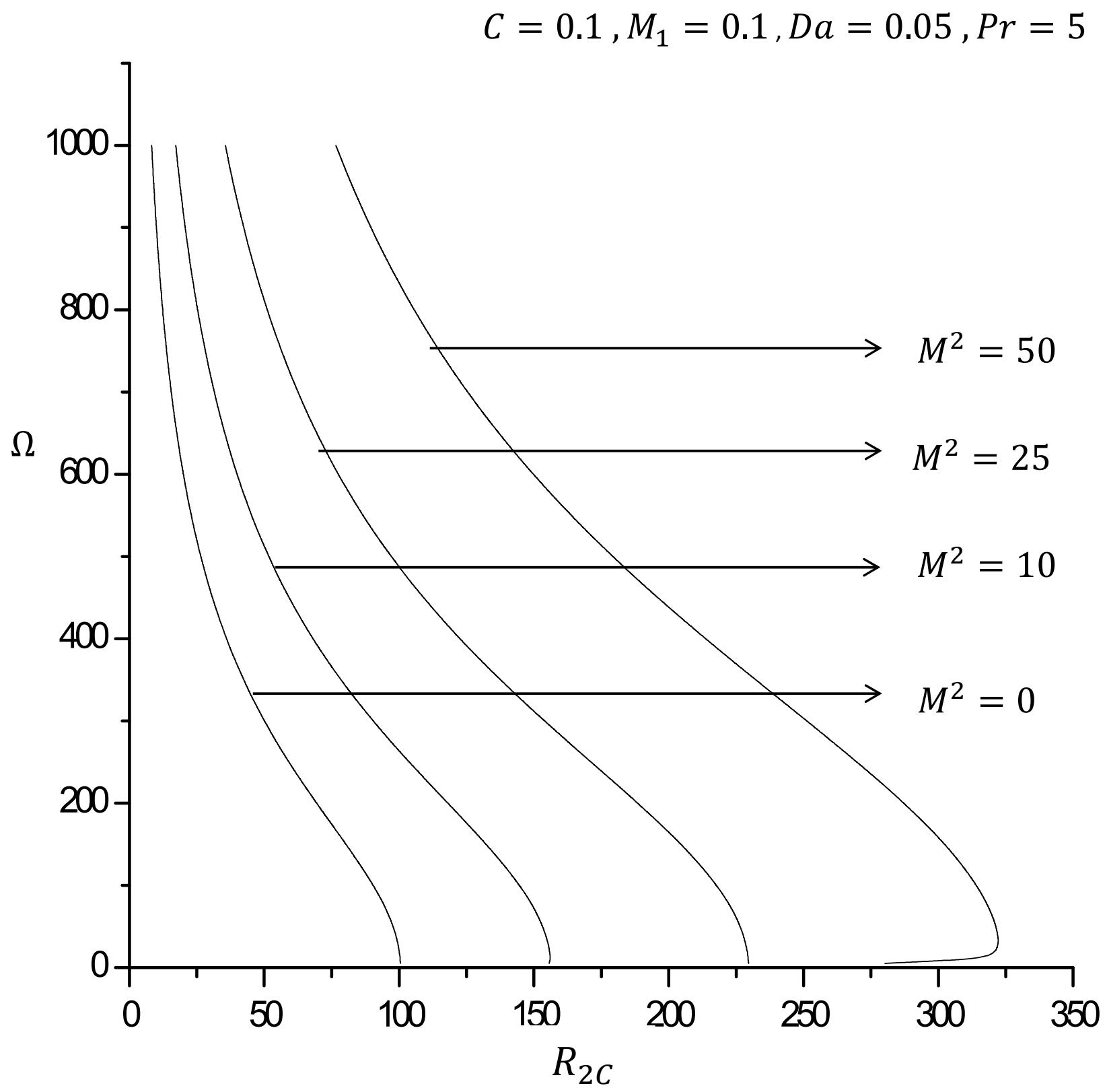

Fig 3: The plot of correction Rayleigh number $R_{2 C}$ vs frequency of modulation $\Omega$ for different values of Hartmann number $M^{2}$. 


$$
C=0.1, M^{2}=10, \operatorname{Pr}=5, M_{1}=0.1
$$

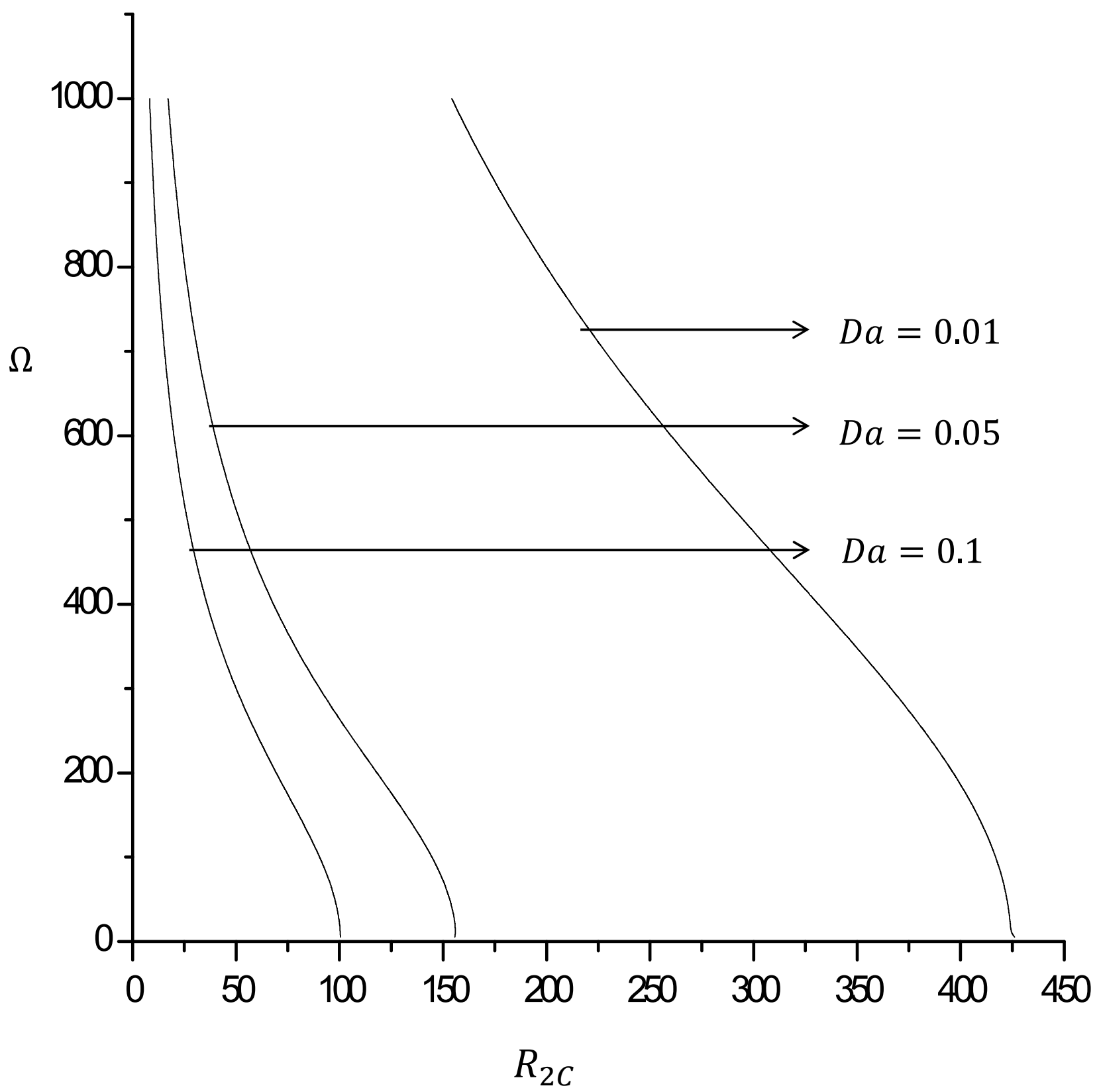

Fig 4: The plot of correction Rayleigh number $R_{2 C}$ vs frequency of modulation $\Omega$ for different values of Darcy number $D a$. 


$$
C=0.1, M^{2}=10, D a=0.05, \operatorname{Pr}=5
$$

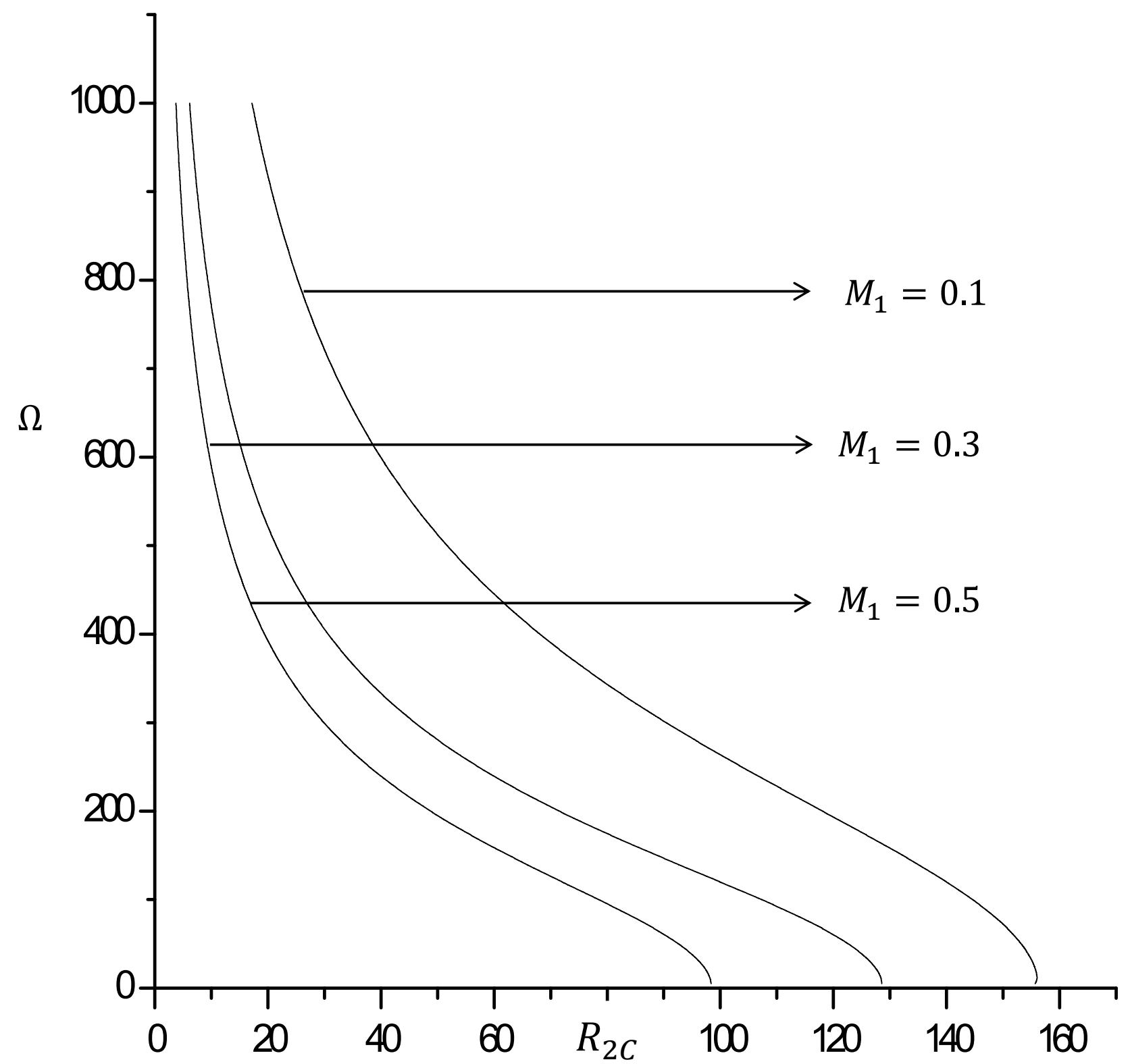

Fig 5: The plot of correction Rayleigh number $R_{2 C}$ vs frequency of modulation $\Omega$ for different values of Porous parameter $M_{1}$. 


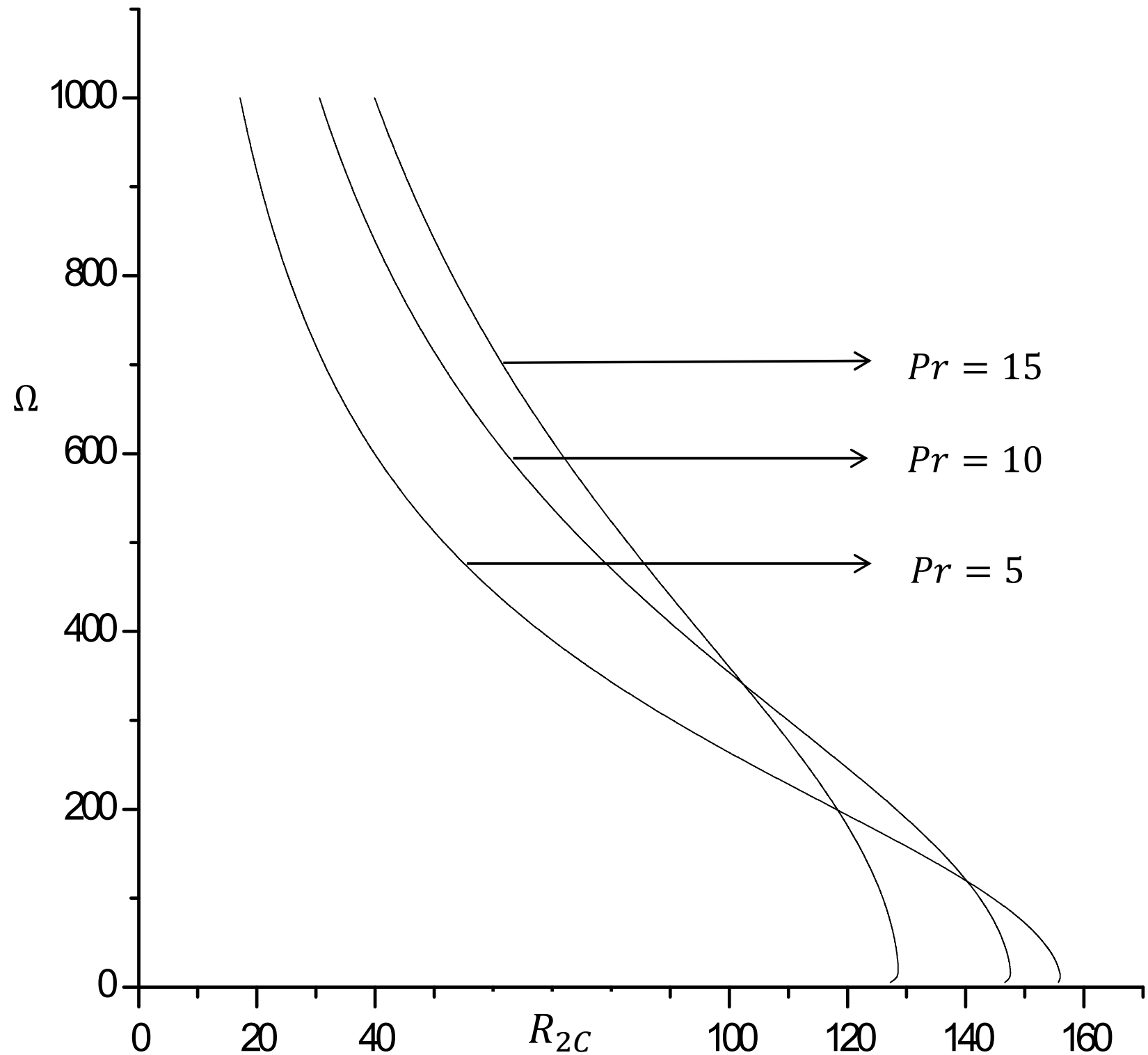

Fig 6: The plot of correction Rayleigh number $R_{2 C}$ vs frequency of modulation $\Omega$ for different values of Prandtl number $P r$. 


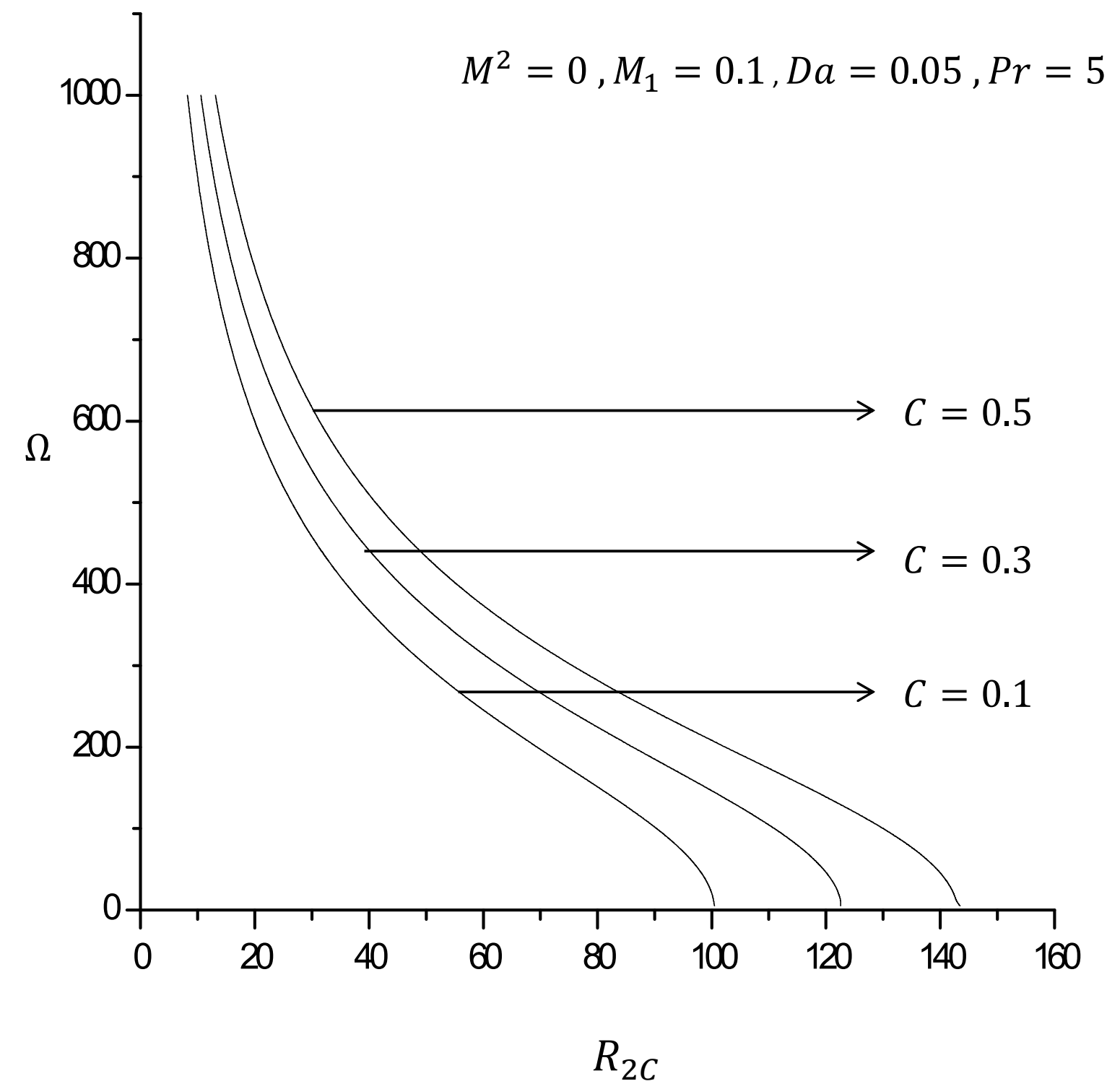

Fig 7: The plot of correction Rayleigh number $R_{2 C}$ vs frequency of modulation $\Omega$ for different values of Couple stress parameter $C$. 


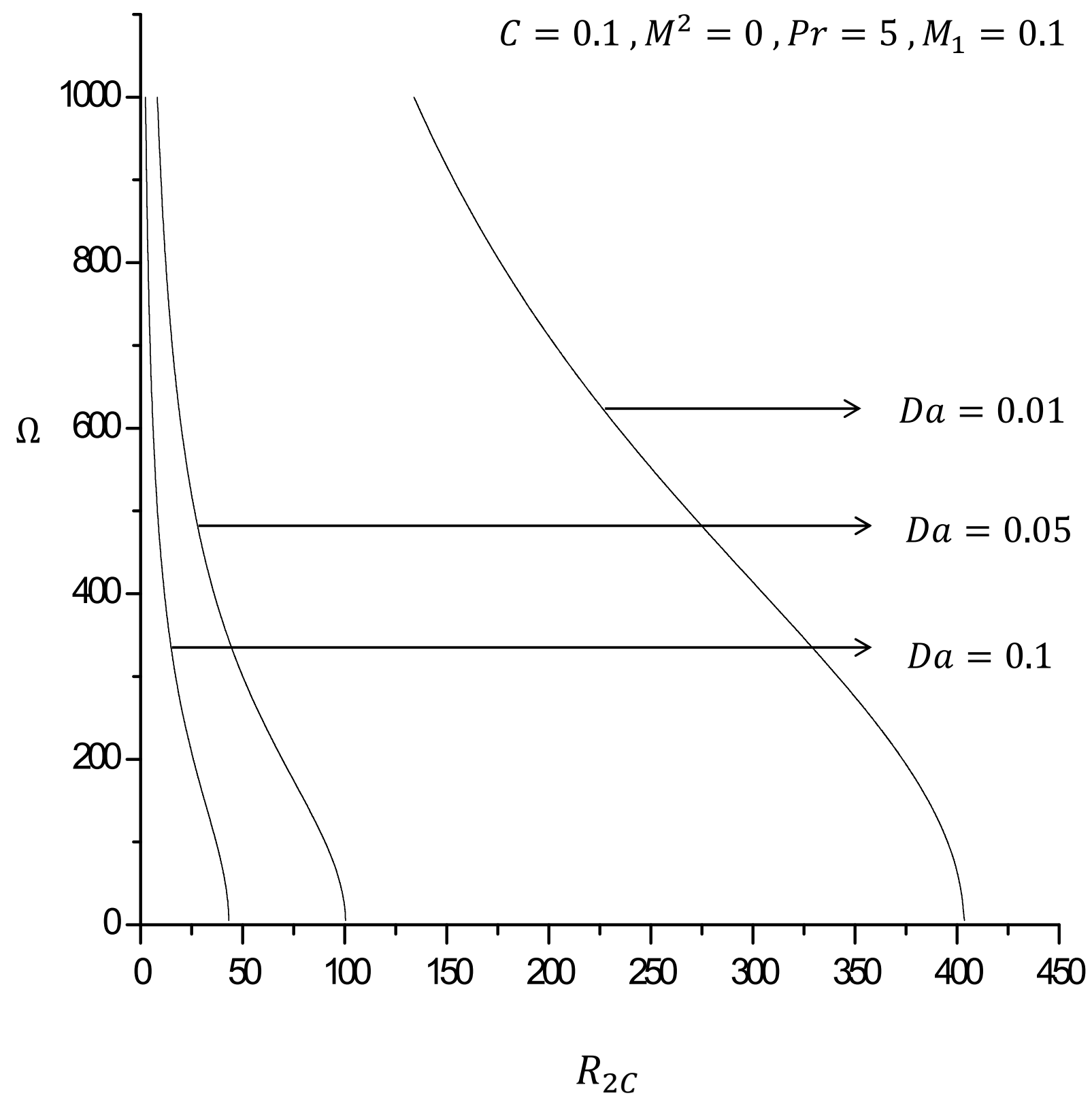

Fig 8: The plot of correction Rayleigh number $R_{2 C}$ vs frequency of modulation $\Omega$ for different values of Darcy number $D a$. 


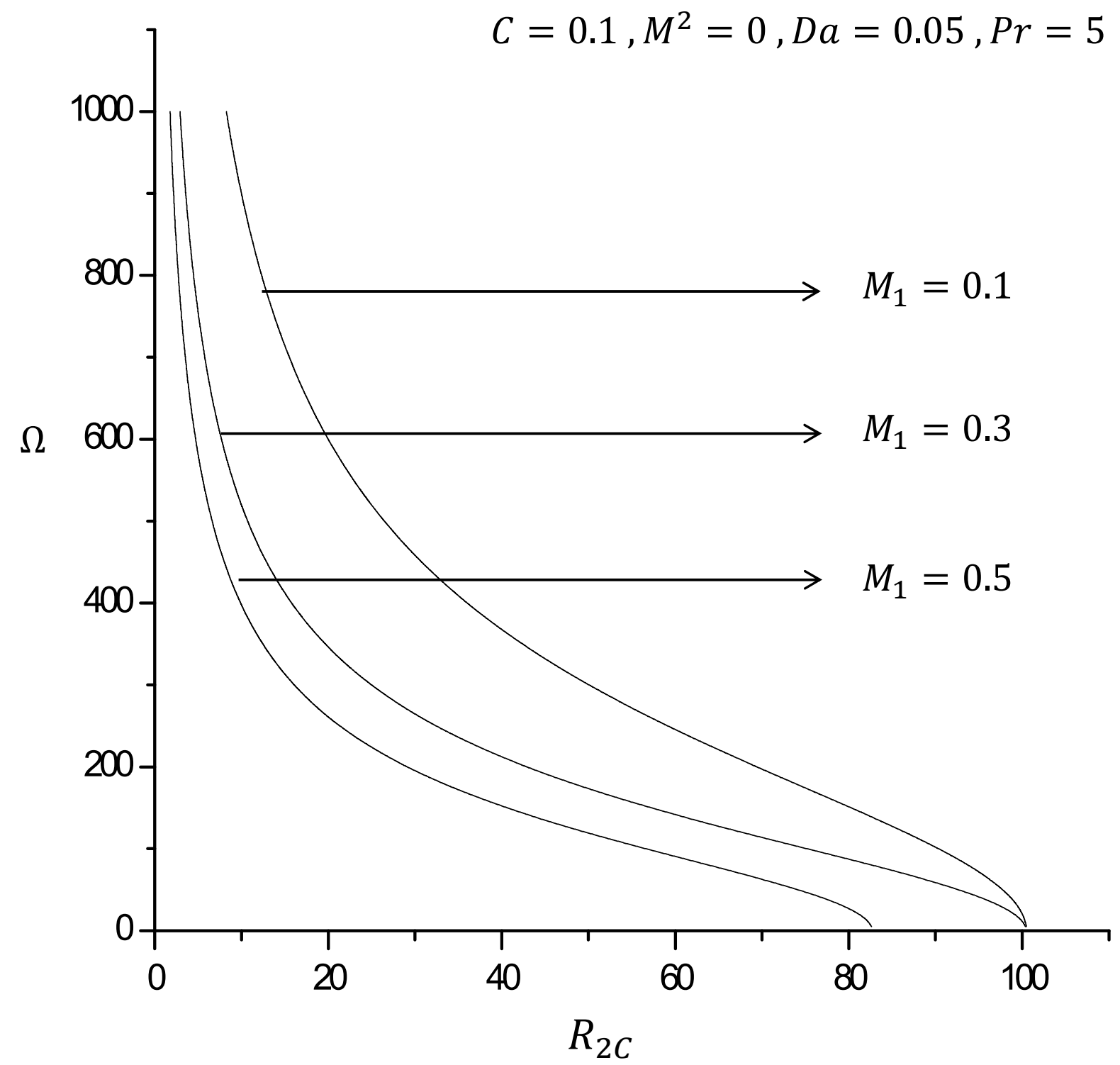

Fig 9: The plot of correction Rayleigh number $R_{2 C}$ vs frequency of modulation $\Omega$ for different values of Porous parameter $M_{1}$. 


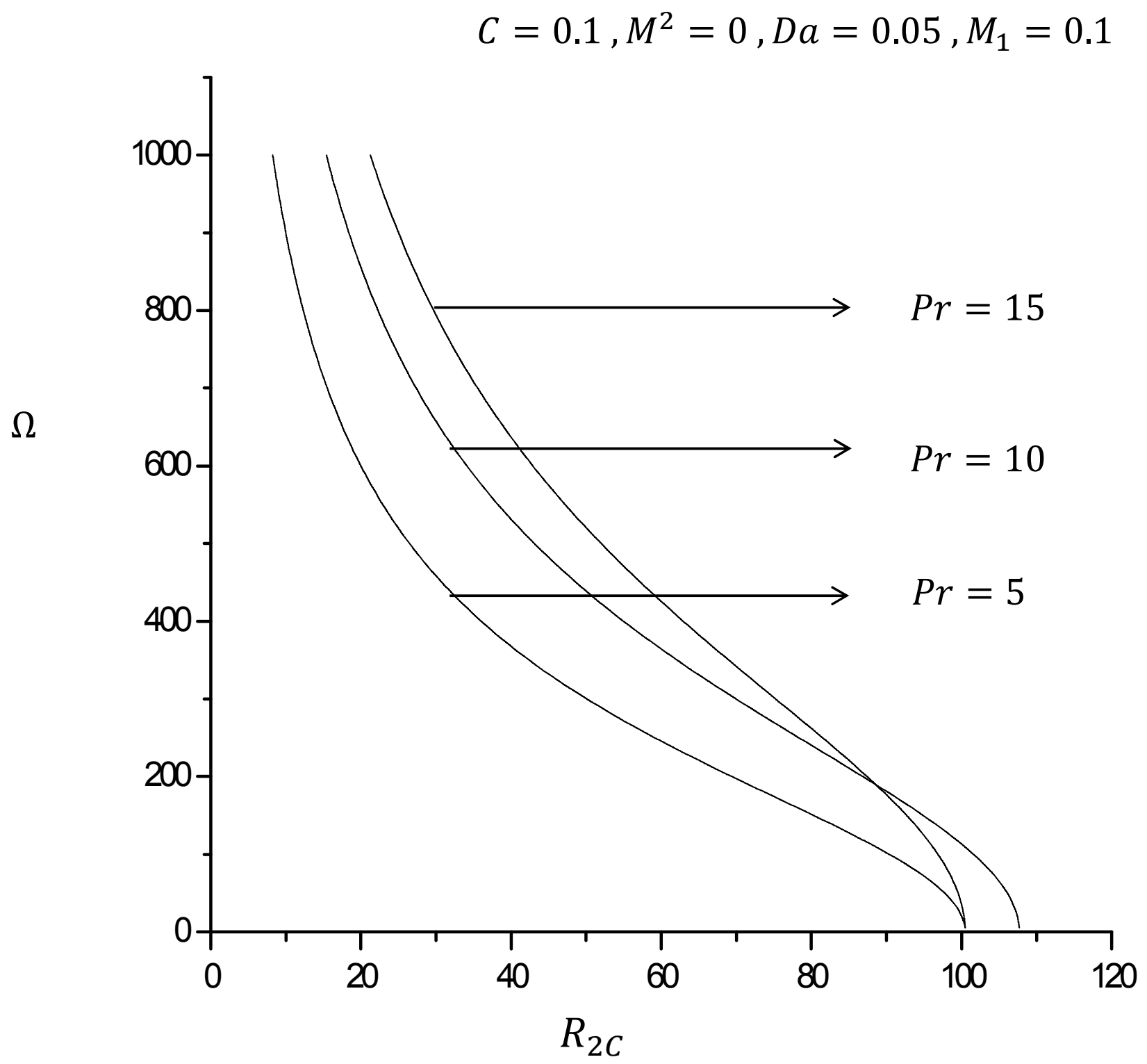

Fig 10: The plot of correction Rayleigh number $R_{2 C}$ vs frequency of modulation $\Omega$ for different values of Prandtl number $P r$.

\section{CONCLUSION}

From the study we conclude that the gravity modulation or g-jitters leads to delay in convection. Also the results suggest that instead of taking electrically non-conducting fluid it is better to consider electrically conducting fluid with weak electrical conductivity as this ensures a stable environment in the presence of a magnetic field.

\section{BIBLIOGRAPHY}

[1] I.S. Shivakumara et al. "Effect of Non-Uniform Temperature Gradients on the Onset of Convection in a Couple-Stress FluidSaturated Porous Media." Journal of applied fluid mechanics, vol. 5, pp.49-55, 2012.

[2] M.K. Veera et al. "Hall effects on steady hydro magnetic flow of a couple stress fluid through a porous medium in a rotating parallel plate channel.” Bull. Pure Appl. Sci. Sect. E Math. Stat., pp. 183192, 2010

[3] M.S. Malashetty and D. Basavaraja. "Rayleigh- Bénard convection subject to time dependent wall temperature/gravity in fluid-saturated 
anisotropic porous medium.” Int. J. Heat Mass Transfer, Vol.38, 2002.

[4] P.G. Siddheshwar and A. Abraham. "Effect of time-periodic boundary temperatures/body force on Rayleigh- Bénard convection in a ferromagnetic fluid." Acta Mech., 131-150, 2003.।

[5] P.G. Siddheshwar and S. Pranesh. "Effect of temperature/gravity modulation on the onset of magneto-convection in weak electrically conducting fluids with internal angular momentum." Int. J. Magn. Magn. Mater, pp. 159-176, 1999.

[6] P.G. Siddheshwar and S. Pranesh. "Effect of temperature/gravity modulation on the onset of magneto-convection in electrically conducting fluids with internal angular momentum." Int. J. Magn. Magn. Mate, pp. 153-162, 2000.

[7] P.G. Siddheshwar and S.Pranesh. "An analytical study of linear and non-linear convection in Boussinesq-Stokes suspensions." Int. J. Non-Linear Mech, pp. 165-172, 2004.

[8] Pranesh S et al. (2014). "Linear and Weakly Non-Linear Analyses of Gravity Modulation and Electric Field on the onset of RayleighBénard Convection in a Micropolar Fluid." Journal of Advances in Mathematics 9(3), pp. 2057-2082.

[9] V.K. Stokes. "Couple stress in fluids." Phys. Fluids, pp. 1079-1715, 1966.

[10] V.K. Stokes. "Theories of fluids with microstructure." Springer, New York, 1984. 